\title{
AVALIÇÃO DA RESPOSTA IMUNE DO HEMIPTERA Rhodnius prolixus FRENTE À INFECÇÃO COM O FUNGO ENTOMOPATOGÊNICO Metarhizium anisopliae
}

\author{
Marcelle de Queiroz Guimarães ${ }^{1}$ \\ Anderson Ribeiro ${ }^{2}$ \\ Adriano Rodrigues de Paula ${ }^{3}$ \\ Simone Azevedo Gomes ${ }^{4}$ \\ Richard lan Samuels ${ }^{5}$ \\ Rodrigo Nunes da Fonseca ${ }^{6}$ \\ Raquel Juliana Vionette do Amaral ${ }^{7}$ \\ Marcos Henrique Sorgine ${ }^{8}$ \\ Jorge Moraes ${ }^{9}$ \\ Flávia Borges Mury ${ }^{10}$
}

Resumo: O hemípetra Rhodnius prolixus é vetor da doença de chagas. O fungo Metarhizium anisopliae apresenta patogenicidade e virulência contra várias espécies de insetos e vêm sendo utilizado como inseticida biológico contra diferentes pragas e vetores. Dessa forma, o objetivo do presente trabalho foi avaliar o perfil da resposta imune de R. prolixus após infecção com fungo entomopatogênico M. anisopliae. Para isso foram utilizadas fêmeas adultas em jejum ou alimentadas com sangue de coelho e, posteriormente, infectado com o fungo M. anisopliae. A modulação da resposta imune foi estudada pela análise da abundância de RNAm codificando Dorsal e Cactus (via Toll), Relish (via IMD), Eigher (ortólogo de TNF), STAT e SOCS (via Jack-STAT), bem como Defensina e Lisozimas (LysA, LysB) (peptídeos antimicrobianos) por RT-qPCR. O EF-1 (Elongation Factor-1) foi utilizado como gene de referência. Os resultados preliminares mostraram que a infecção por fungo foi capaz de reduzir a taxa de eclosão de $\mathrm{R}$. prolixus em $40 \%$. Foi evidenciado que as Vias Toll e IMD estão envolvidas na defesa imune contra M. anisopliae.

Palavras-chave: Rhodnius prolixus; sistema imune; Metarhizium anisopliae.

\footnotetext{
1 Universidade Federal do Rio de Janeiro - Núcleo de Ecologia e Desenvolvimento Socioambiental de Macaé Laboratório Integrado de Bioquímica Hatisaburo Masuda, Av. São Jose do Barreto, 724, Macaé-RJ, Brasil. E-mail: marcelleqguimaraes@hotmail.com

2 Universidade Estadual do Norte Fluminense - Centro de Ciências e Tecnologias Agropecuárias - Laboratório de Entomologia e Fitopatologia, Av. Alberto Lamego, 2000, Campos dos Goytacazes-RJ, Brasil. E-mail: anderson.ribeirorj@yahoo.com.br.

3 Universidade Estadual do Norte Fluminense - Centro de Ciências e Tecnologias Agropecuárias - Laboratório de Entomologia e Fitopatologia, Av. Alberto Lamego, 2000, Campos dos Goytacazes-RJ, Brasil. E-mail: biodepaula@yahoo.com.br.

4 Universidade Estadual do Norte Fluminense - Centro de Ciências e Tecnologias Agropecuárias - Laboratório de Entomologia e Fitopatologia, Av. Alberto Lamego, 2000, Campos dos Goytacazes-RJ, Brasil. E-mail: simoneazgomes@yahoo.com.br

5 Universidade Estadual do Norte Fluminense - Centro de Ciências e Tecnologias Agropecuárias - Laboratório de Entomologia e Fitopatologia, Av. Alberto Lamego, 2000, Campos dos Goytacazes-RJ, Brasil. E-mail: richard@uenf.br

6 Universidade Federal do Rio de Janeiro - Núcleo de Ecologia e Desenvolvimento Socioambiental de Macaé Laboratório Integrado de Ciências Morfofuncionais, Av. São Jose do Barreto, 724, Macaé-RJ, Brasil. E-mail: rodrigo.nunes.da.fonseca@gmail.com

7 Universidade Federal do Rio de Janeiro - Instituto de Bioquímica Médica, Cidade Universitária, CCS, Bloco H, Ilha do Fundão, Rio de Janeiro-RJ, Brasil. E-mail:raqueljuvia@yahoo.com.br

${ }^{8}$ Universidade Federal do Rio de Janeiro - Instituto de Bioquímica Médica, Cidade Universitária, CCS, Bloco H, Ilha do Fundão, Rio de Janeiro-RJ, Brasil. E-mail:sorgine@bioqmed.ufrj.br

9 Universidade Federal do Rio de Janeiro - Núcleo de Ecologia e Desenvolvimento Socioambiental de Macaé Laboratório Integrado de Bioquímica Hatisaburo Masuda, Av. São Jose do Barreto, 724, Macaé-RJ, Brasil. Email:jlcmoraes@yahoo.com.br

10 Universidade Federal do Rio de Janeiro - Núcleo de Ecologia e Desenvolvimento Socioambiental de Macaé Laboratório Integrado de Bioquímica Hatisaburo Masuda, Av. São Jose do Barreto, 724, Macaé-RJ, Brasil. Email:fbmury@macae.ufrj.br
} 